\title{
Leptomeningeal Dissemination of Intraventricular Rhabdoid Meningioma: Imaging Findings
}

\author{
Ihsan YUCE ${ }^{1}$, Suat EREN ${ }^{1}$, Akin LEVENT ${ }^{1}$, Mecit KANTARCI ${ }^{1}$, Ali KURT² ${ }^{2}$, Onder Hilmi OKAY³ \\ ${ }^{1}$ Ataturk University Medical School, Department of Radiology, Erzurum, Turkey \\ ${ }^{2}$ Regional Training and Research Hospital, Department of Pathology, Erzurum, Turkey \\ ${ }^{3}$ Regional Training and Research Hospital, Department of Neurosurgery, Erzurum, Turkey
}

\section{ABSTRACT}

A 20-year-old male patient was admitted to our clinic with a 1-year history of headache. The patient's systemic-neurological examination and laboratory findings were normal. Computed tomography and magnetic resonance imaging were performed. Imaging findings showed calcified intraventricular mass and subependymal and gyral nodular lesions. There was a slight increase in ventricular volume. Surgical treatment was performed. Pathological specimens revealed the diagnosis of rhabdoid meningioma. Leptomeningeal dissemination refers to diffuse seeding of the leptomeninges by tumor metastases. To our knowledge, leptomeningeal dissemination of intraventricular rhabdoid meningioma is very rare in the literature. We aimed to discuss imaging findings and differential diagnosis of leptomeningeal dissemination of rhabdoid meningioma.

KEYWORDS: Rhabdoid meningioma, Leptomeningeal dissemination, Headache

\section{INTRODUCTION}

Meningiomas are the most common non-glial primary brain tumor and account for $15 \%-20 \%$ of all primary brain tumors. They are the most common intracranial extra-axial neoplasms (1). Rhabdoid meningioma (RM) was first described in 1998 as an unusual variant of meningiomas (5). It has an increased proliferative activity and is classified as a World Health Organization (WHO) grade III meningioma (5). This case report shows imaging findings and differential diagnosis of leptomeningeal dissemination of intraventricular RM.

\section{CASE REPORT}

A 20-year-old male patient was referred to our clinic with a 1 -year history of headache. The patient's clinical examination and laboratory findings were normal. Computed tomography (CT) and magnetic resonance imaging (MRI) were performed. CT images showed intraventricular mass and calcified subependymal nodules (Figure 1A). MRI study showed contrast enhancing gyral and subependymal nodules bilaterally in cerebral hemispheres and lateral ventricles due to leptomeningeal dissemination (Figure 1B-D). Diffusion weighted images (DWI) showed peripheral restricted diffusion in the mass (Figure 2A, B). He was operated on via left transcortical transventricular approach. A soft, well demarcated, grey tumor tissue demonstrating slight infiltration to the ependymal layer of the anterior horn of the left lateral ventricle was gross totally removed. There was no postoperative complication and he was discharged on the $7^{\text {th }}$ postoperative day. Pathological specimens revealed the diagnosis of RM (Figure 3A, B).

\section{DISCUSSION}

$\mathrm{RM}$ is a rare subtype of meningioma, classified as WHO grade III with a poor prognosis. Anaplastic meningiomas represent $2-3 \%$ of all meningiomas (4). According to the literature, most RMs behave aggressively and have a very poor prognosis (5). It is important to recognize rhabdoid morphology in a menin- 

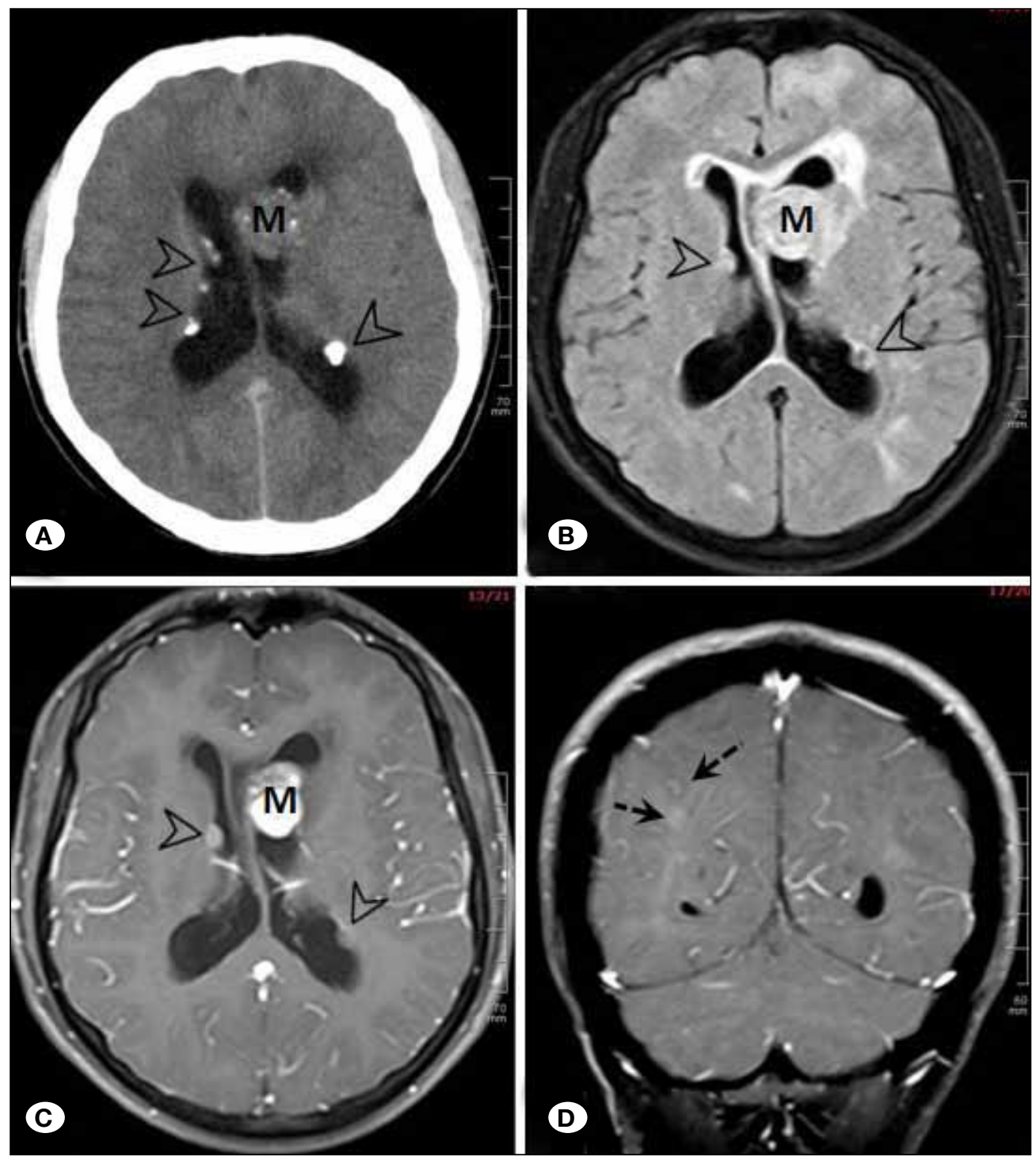

Figure 1: Non-contrast axial CT image (A), FLAIR MR image (B), contrast enhanced T1 weighted axial (C) and coronal (D) MR images show intraventricular mass in the left lateral ventricle and bilateral subependymal (arrowheads) and gyral nodular (dashed arrows) lesions associated with leptomeningeal dissemination of tumor. $\mathbf{M}$ : mass.
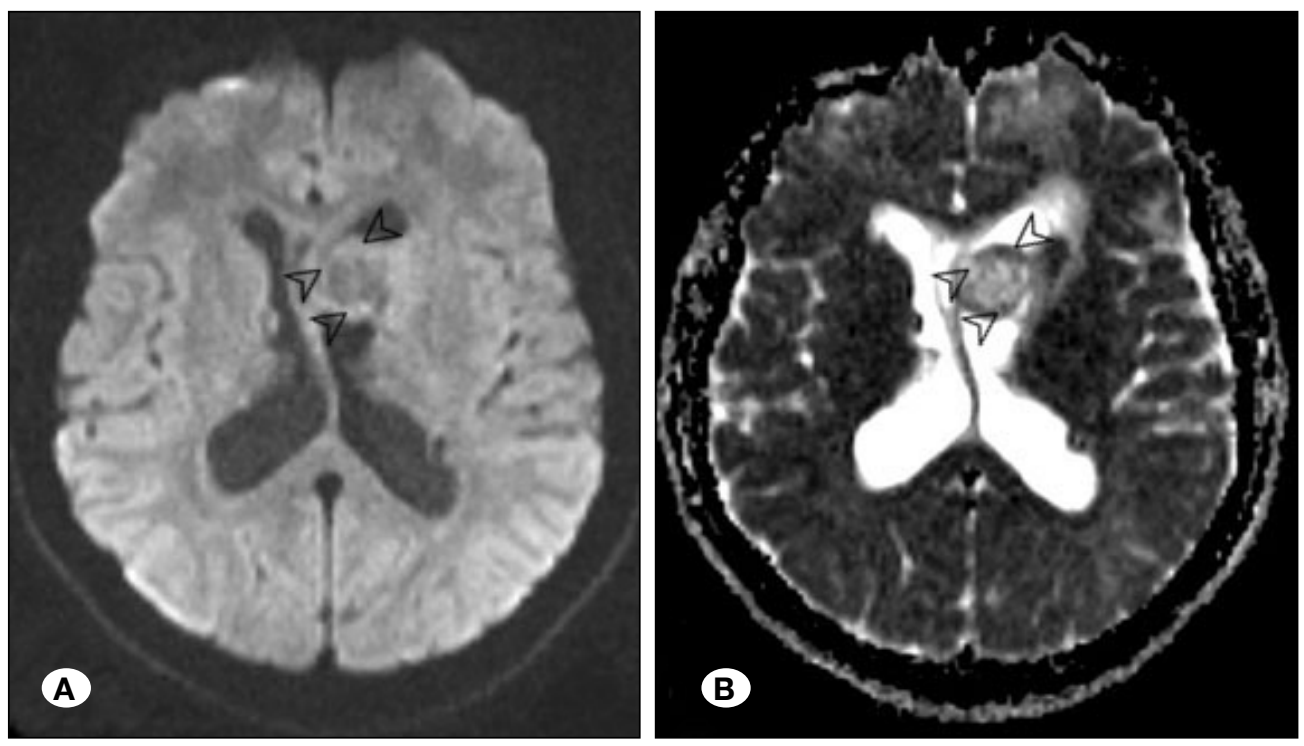

Figure 2: DWI $(b=1000)$ (A) and ADC mapping (B) images show clear peripheral restricted diffusion in the rhabdoid meningioma (arrowheads). 

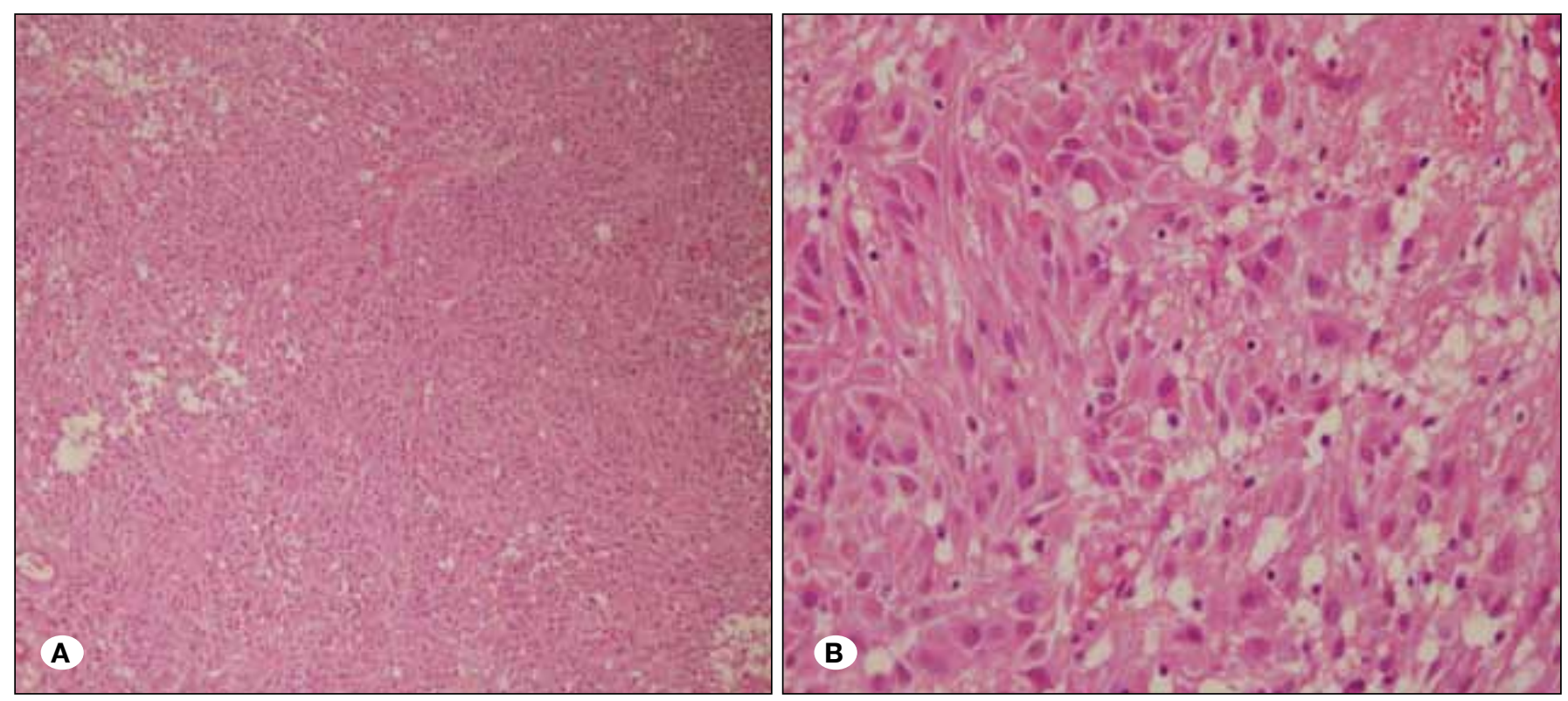

Figure 3: Histopathology of rhabdoid meningioma. H\&Ex100 (A), H\&Ex400 (B).

gioma early to help in both the diagnosis and understanding of its clinical course.

The differential diagnosis for such intraventricular masses as in our case should include ependymoma, subependymoma, central neurocytoma, subependymal giant cell astrocytoma, choroid plexus papilloma and carcinoma, metastasis and meningioma (6).

Leptomeningeal metastasis is a common complication of cancer. It is often difficult to diagnose, but early diagnosis and aggressive treatment can prevent irreversible neurologic deficits. It is also very important for neurosurgeons to have knowledge about this condition in advance. Diagnosis is usually established by the demonstration of malignant cells in the cerebrospinal fluid (CSF) or by the presence of enhancing tumor nodules on cranial or spinal MRI (3).

The most common locations for meningiomas (in descending order) are the parasagittal dura, convexities, sphenoid wing, cerebellopontine angle cistern, olfactory groove and planum sphenoidale. Intraventricular meningiomas are rare, constituting only $0.7 \%$ of all meningiomas (13). Still, this tumor is one of the most common intraventricular neoplasms in the adult population, and, in some reports, a meningioma is the most common atrial mass to manifest in an adult patient. Most intraventricular meningiomas occur in patients older than 30 years and the peak age is between 30 and 60 years (7). Females are more likely to be affected, with a 2:1 ratio $(6,7)$. In the pediatric population, almost one-fifth of all meningiomas occur within the ventricular system (7). Patients with intraventricular meningiomas present with symptoms related to increased intracranial pressure (headache, nausea, vomiting), contralateral sensory or motor deficits, and homonymous hemianopsia. The trigone of the lateral ventricle is the most common site, with a slight majority of the reported cases being located on the left side $(6,11,12)$.
On CT images, intraventricular meningiomas manifest with an appearance similar to that of other intracranial meningiomas: a well-defined globular mass that demonstrates hyperattenuation compared with the brain parenchyma. Calcification is common (50\% of cases) (6). On MRI scans, those originating within the ventricles characteristically are isointense to hypointense compared with gray matter with T1-weighted imaging and isointense to hyperintense with T2-weighted imaging (6). Atypical and malignant subtypes may show greater than expected restricted diffusion although recent work suggests that this is not useful in prospectively predicting histological grade (10).

The histological pattern of tumour was characterized by the presence of rhabdoid cells which were large, round or ovoid with abundant eosinophilic cytoplasm and prominent, vesicular, often eccentrically located nuclei with prominent paranuclear nucleoli (Figure 3A, B). Meningothelial cells were wrapped around small blood vessels. The rhabdoid cells stain diffusely for S 100 and Vimentin and show focal expression of epithelial membrane antigen. Mitoses were seen and $\mathrm{Ki}$ 67 positive cells were $20 \%$. Progesterone and GFAP were negative.

Complete surgical resection including the dural attachment is a preferred therapeutic approach for all meningiomas, including those that are malignant. As delineated in National Comprehensive Cancer Network (NCCN) Guidelines, surgery is the first line of treatment for all symptomatic and for large asymptomatic meningiomas (8). With the advancements in modern imaging techniques, microsurgery, and imageguided intraoperative approaches, this can be achieved in most tumors at accessible locations, minimizing the damage to normal brain tissue. However, malignant meningiomas often cannot be completely resected. Subtotal resection is then combined with radiation therapy. The extent of surgery is balanced with minimizing neurological deficits possibly 
caused by resection. Complete resection is usually attempted for tumors of the convexity, olfactory groove, anterior third of the sagittal sinus and some tentorial and posterior fossa tumors. In addition, as malignant meningiomas are highly vascular tumors, preoperative embolization can make them more resectable. It is most commonly applied to skull base meningiomas, a day prior to surgery (2). As with other modalities of systemic treatment, chemotherapy has been mainly used for recurrent tumors after the surgical and radiation options have been exhausted. Postoperative management should include following: prevention of seizures, cerebral edema and deep venous thrombosis and application of radiation therapy. Routine checks for imaging should be performed by CT or MRI.

WHO grade III tumors are significantly more likely to be invasive and show local recurrence following the initial treatment (even gross total resection). Prognosis worsens with poorer differentiation of the tumors. A high mitotic rate is significant prognostic factor, as well as poor Karnofsky performance status (9). A study reported that 3 and 5 -year recurrence-free survival rates were 50 and $29 \%$, respectively for patients with anaplastic lesions (WHO grade III) (14).

In conclusion, rhabdoid meningiomas are rare and their leptomeningeal dissemination is very rare (only a few cases in the literature). CT and MRI findings are very important for early diagnosis of an intraventricular mass and subependymal nodules.

\section{- REFERENCES}

1. Buetow MP, Buetow PC, Smirniotopoulos JG: Typical, atypical, and misleading features in meningioma. Radiographics 11:10871106, 1991

2. Carli DF, Sluzewski M, Beute GN, van Rooij WJ: Complications of particle embolization of meningiomas: Frequency, risk factors and outcome, AJNR Am J Neuroradiol 31:152-154, 2010

3. DeAngelis LM, Boutros D: Leptomeningeal metastasis. Cancer Invest 23:145-154, 2005
4. Johnson MD, Atkinson JB: Central nervous system tumors. In: Weidner N, Cote RJ, Suster S, Weiss LM (eds). Modern Surgical Pathology. Vol: 2, 2nd ed. Elsevier 2009: 1984-2038

5. Kepes JJ, Moral LA, Wilkinson SB, Abdullah A, Llena JF: Rhabdoid transformation of tumor cells in meningiomas: A histologic indication of increased proliferative activity: Report of four cases. Am J Surg Pathol 22:231-238, 1998

6. Koeller KK, Sandberg GD: From the archives of the AFIP. Cerebral intraventricular neoplasms: Radiologic-pathologic correlation. Radiographics 22:1473-1505, 2002

7. Lang I, Jackson A, Strang F: Intraventricular hemorrhage caused by intraventricular meningioma: CT appearance. AJNR Am J Neuroradiol 16: 1378-1381, 1995

8. National Comprehensive Cancer Network (NCCN): NCCN Guidelines. TM Version 2. Central Nervous System Cancers, MENI-1, http://www.nccn.org/professionals/physician_gls/f_ guidelines.asp\#site, 2011

9. Pasquier D, Bijmolt $S$, Veninga T, Rezvoy N, Villa S, Krengli M, Weber DC, Baumert BG, Canyilmaz E, Yalman D, Szutowicz E, Tzuk-Shina T, Mirimanoff RO: Atypical and malignant meningioma: outcome and prognostic factors in 119 irradiated patients. A multicenter, retrospective study of the rare cancer network. Int $\mathrm{J}$ Radiat Oncol Biol Phys 71:1388-1393, 2008

10. Sanverdi SE, Ozgen B, Oguz KK, Mut M, Dolgun A, Soylemezoglu $F$, Cila $A$ : Is diffusion weighted imaging useful in grading and differentiating histopathological subtypes of meningiomas? Eur $\mathrm{J}$ Radiol 81: 2389-2395, 2012

11. Sekerci Z, Oral N, Ugurluglu O, Colpan E, Ugur A: Evaluation of forty-five atypical and malignant meningioma cases: Over the 12-years follow-up period. Turk Neurosurg 14:12-20, 2004

12. Sgouros S, Walsh A, Barber P: Intraventricular malignant meningioma in a 6-year-old child. Surg Neurol 42:41-45,1994

13. Strenger $S$, Huang $Y$, Sachdev V: Malignant meningioma within the third ventricle: A case report. Neurosurgery 20:465-468, 1987

14. Yang SY, Park CK, Park SH, Kim DG, Chung YS, Jung HW: Atypical and anaplastic meningiomas: Prognostic implications of clinicopathological features. J Neurol Neurosurg Psychiatry 79: 574-580, 2008 